\title{
Some characteristics of entrepreneurship in medicine. The digitalization of the medical system in Romania
}

\author{
Irina Dijmarescu ${ }^{1,2}$ \\ 1 "Carol Davila" University of Medicine and Pharmacy, Bucharest, Romania \\ ${ }^{2}$ Department of Pediatrics, "Grigore Alexandrescu" Emergency Children's Hospital, Bucharest, \\ Romania
}

\begin{abstract}
Entrepreneurship and medicine are in continuous growth alongside, and even if they appear to be completely dif-
ferent fields, entrepreneurship in medicine is becoming of remarkable interest, further highlighted by the COVID-19
pandemic. A main reason for this is its ability to use digital technologies, which are meant to improve healthcare.
Applying digitalization in healthcare includes not only computer and database use (cloud computing, big data), but
more complex methods such as robotics, automation, internet of things, artificial intelligence and, not lastly, col-
laboration platforms. Some shortcomings in respect to digitalization can be identified in the Romanian healthcare
system and these may impact public health. Digitalization may contribute fundamentally to the improvement of
public health by increasing performance. However, in the context of the COVID-19 pandemic, other ailments that
burden the healthcare system should not be neglected (cardiovascular diseases, malignancies, etc.).
Keywords: medicine, entrepreneurship, digital technologies
\end{abstract}
\section{INTRODUCTION}

Entrepreneurship is currently of remarkable interest in medicine, both because of the COVID-19 crisis and of its ability to use digital technologies in order to improve public health. Even though they appear to be completely different fields, medicine and entrepreneurship have common elements, and there are bridges and factors that enhance each other. Healthcare personnel is trained to solve problems in order to establish a correct diagnosis, facing uncertainty, sometimes based on information that cannot be correlated, is incomplete or in continuous change. Among the problems that need to be managed is the cost of the necessary tests and procedures.

For the implementation of entrepreneurship in medicine it is necessary for the individual who constructs the business plan to possess not only an innovative idea, but also adequate entrepreneurship abilities. Entrepreneurship in general, in order to be applied in medicine, needs to be adjusted and cus- tomized. The domain of action focuses on the health of the human being - both individual health and public health.

Entrepreneurship in medicine implies a manager who wishes to take the risk of starting a business in healthcare, using personal funds or a loan (from banking or non-banking financial institutions). Healthcare entrepreneurship may be encouraged by corrective subsidies - the business is funded by the local or central public authority in order to enhance the benefits provided by the implemented business plan. It is necessary for the initiative to be beneficial for the society or at least for a large number of people, generating positive externalities - short, medium or long term marginal external benefits (healthcare services provided are free of charge).

The cost benefit analysis and SWOT analysis (strengths, weaknesses, threats and opportunities) are of major importance in order to obtain funding. Usually, estimating the costs for a lucrative activity is a more accurate process in comparison to assessing the 
magnitude of the benefits observed in a population which is difficult to establish, especially when short-, medium- and long-term effects should be taken into consideration (1).

The manager should have the ability, on his own or in partnership, to implement and develop the innovative idea on the market. A team which will be able to apply the respective project and procure funding for attaining the established objectives is also needed.

The healthcare entrepreneur should ensure services are available based on a schedule that may not always be constant - emergency, intermittent or permanent care might be needed. As means to provide quality healthcare it is necessary for the purveyor to be scientifically updated (state-of-the-art knowledge). Because medicine requires collusion with other fields of activity, including the economic one, it is considered to be a multidisciplinary topic

In general, entrepreneurship in medicine has been considered an exception rather than a rule, but COVID-19 crisis has driven it towards applying digital technologies in order to avoid inter human contact.

Digitalization is the fourth state of the industrial revolution. Its interrelated and diverse components are increasingly used in an higher number of fields and activities, and it may be associated to disruptive technologies. Disruptive technologies are, in the broadest sense, those which transform axioms and paradigms, and in a narrow sense, they are meant to completely change the rules in all domains, including the medical one. As in other fields, in medicine digitalization is increasingly used. It is a topic of perspective, more and more spread in healthcare. A complex transfer of digital technologies becomes apparent, which materializes in different components of digitalization. These impact prevention, treatment and post therapeutic monitoring (2).

Applying digitalization in healthcare includes not only computer and database use (cloud computing, big data), but more complex methods such as robotics, automation, internet of things, artificial intelligence and, not lastly, collaboration platforms.

The use of collaboration platforms is of major benefit because solving a medical problem (establishing a diagnosis, applying optimal treatment, etc.) becomes available based on the experience of several professionals in the field, overcoming the geographical barrier and reducing the reaction time.

Internet of things comprises of a series of sensor and software integrated devices, which are wireless interconnected, devices that are able to collect, provide, store and receive information in a network, without the need of inter human or human to com- puter interaction. This kind of system has numerous benefits on public health, highlighted even more because of the COVID-19 pandemic. Internet of things has the ability to facilitate data collection and analysis and predict the occurrence of certain ailments, as well as the possibility to establish a diagnosis and contribute to establishing the treatment and monitoring patients both in outpatient and inpatient facilities. Globally, implementation of needed policies for launching medicine-based technologies is of interest. This structure responds to certain needs in the context of a higher prevalence of chronic diseases, with high impact on the healthcare system. Hence, each individual may personally manage different medical issues using innovations, subsequently reducing the burden on the healthcare facilities (3).

Big data was first introduced in the $90 \mathrm{~s}$ and it is estimated that its major use in the following years will be in the medical field (4). The concept of big data is characterized by the acceleration of collecting, processing and analyzing data. A higher volume of data is available at a certain point, with increased variability (originating from multiple sources). Data of interest may be divided in different categories molecular, imaging or digital data, or data measured by applying sensors. Higher volume of more variable information is collected, and this is because electronic health records are widely available worldwide $(5,6)$. Big data technologies and artificial intelligence are used in numerous fields of medicine, approaching both clinical aspects - in cardiology (management of hypertension or cardiac failure), dermatology, somnology (monitoring sleep apnea) etc. - and of medical research $(4,7-10)$. Data sets may help identify different patterns for certain diseases and may provide solutions for problems that were out of reach to date. But there are still impediments for widely using these technologies, issues regarding international law, public ignorance, personal data protection or security. These aspects delay the progress that may be achieved using big data sets (4).

Even though digital technologies are furthermore applicable in the COVID-19 pandemic context, they appear to have social and economic advantages and a favorable impact on public health. But this does not need to translate into neglecting other ailments with high morbidity and mortality such as obesity, cardiovascular diseases, malignancies, etc. In the pandemic context, the importance of resilience was highlighted. Resilience is defined by a series of protective and salutogenic factors that influence health, response to treatment and ability to heal. Vulnerability and disease risk are lower when the resilience is higher (11). 


\section{DIGITALIZATION OF THE HEALTHCARE SYSTEM IN ROMANIA}

Some shortcomings in respect to digitalization can be identified in the Romanian healthcare system and these may impact public health. Among the evident problems is a high incidence of ailments with high morbidity and mortality such as cardiovascular and malignant diseases, together with a low birth rate. Although the medical insurance system is functional, the sanitary crisis persists (12).

One of the things that may contribute to the improvement of public health is the digitalization of the healthcare system in order to increase performance. This concept may be applied in several areas of the Romanian healthcare system - hospital data bases digitalization, creating electronic health records, use of image processing software, use of digital means for health education in order to promote prevention (primary and secondary prophylaxis), telemedicine, etc. Some of these have been of interest for the authorities for a long time (12). However, in the last year, with the demand of social distancing in the COVID-19 pandemic context, this need has been brought to the front.

Digitalization of the medical system is extremely important both for providing reliable information towards the general population (essential for promoting prevention and early diagnosis, offering a better chance at improvement or healing), as well as for simplifying the interaction between the doctor and the patient. Relieving doctors and patients of medical related activities will bring to the fore the final aim of medicine, healthcare.

All these means are covered by the e-health concept, defined as all communication technologies of interest in providing support for medical information - transmission, record and storage (13). Data storage is possible within an institution, in among several institutions or nationally. It provides doctors with the possibility of clinical documentation and it offers substrate for artificial intelligence (14). A national data base would be of great interest in Romania, and it would improve and facilitate healthcare, providing a detailed medical history (sometimes not available at this point), essential for securing quality healthcare.

E-health applications are diverse and of growing interest, both for doctors and for patients. They aim to provide healthcare services (telemedicine, e-health education, data storage by electronic health records, etc.) or different instruments of great interest in medicine (online conferences, visualization instruments, image analysis, virtual collaboration platforms etc.) (13).
One of the most important branches of digitalization in healthcare is telemedicine, which has been proven of highly importance during the COVID-19 pandemic because of the needed social distancing. Telemedicine offers a bridging solution by means of communication technologies for interaction between doctors or between patients and doctors. It also meets the needs of today's society not only by facilitating social distancing, but also because of the aging society with an increasing number of people with chronic illnesses, morbidity and difficult access to medical care in some areas (few doctors available). Moreover, telemedicine offers the possibility of remote monitoring using portable technologies or smart home devices that may increase the quality of life for some patients. Data collected by remote monitoring is reliable for medical decisions as well (14).

In the last year, because of the international crisis caused by the COVID-19 pandemic, healthcare digitalization was considered a possible solution for approaching disaster medicine. Disasters and destructive events (ex. terrorism, fire, epidemics) are those events which threaten public health or the environment and prevent normal progress of medical activities. They may lead to delayed, improper or lack of access to medical care. Consequently, this burdens the healthcare systems and digitalization may meet an emerging need. Usually, disaster medicine or disaster management doesn't use information or technologies which are specific for healthcare (e-health). Even though communication technology, computerization, widely available internet access and smart devices are achievable, their applicability in disaster medicine is still limited (15).

Healthcare digitalization has been studied in different fields, even before the COVID-19 pandemic. For instance, smart devices (watches, phones) have been used in cardiology and it is estimated that this approach will continue to escalate and will likely change traditional cardiology (16). Another topic of interest is healthcare education in order to improve compliance to treatment and subsequently health status. In this regard, a group of researchers has analyzed weather e-health technologies may improve adherence to treatment and if this plan of action may be used for monitoring patients and identifying the optimal time for intervention in patients with sleep apnea. It was concluded that digitalization for healthcare education would improve therapeutic options acceptance and it might be helpful for monitoring these patients, but there are still unknown aspects regarding what kind of interventions would be appropriate and the best time and duration of action (17).

Digital platforms are business models based on technology that facilitates the interaction among 
agents, thereby achieving a bridge between two nodes that are not connected. Their applicability in healthcare public-private partnerships is essential and may allow complex interaction among partners, expediting the collaboration between public and private sectors. The digital approach comes to support the patient, is patient-centered and is sustainable. Digital technologies are important in the surveillance of communicable diseases (proven aspect in the context of the COVID-19 pandemic), in order to support different forms of healthcare interventions for building up data bases (18).

\section{CONCLUSIONS}

Entrepreneurship and medicine have become connected topics especially by using technology and digitalization. However, medical entrepreneurship has specific features and is meant to improve healthcare. Technology and digitalization are aspects that may represent an important step forward in healthcare and that have been insufficiently approached in Romania to date. The global pandemic in the last year has represented an incentive for digitalization and technology implementation and has highlighted telemedicine and collaboration platforms. Technology and medicine are in continuous growth alongside and thus there is a major benefit towards providing a respite for the healthcare system and healthcare-centered actions.

\section{Acknowledgement}

This paper received financial support through the project entitled "DECIDE - Development through entrepreneurial education and innovative doctoral and postdoctoral research, project code POCU/ $380 / 6 / 13 / 125031$, project co-financed from the European Social Fund through the Operational Program Human Capital 2014 - 2020".

Conflict of interest: none declared

\section{REFERENCES}

1. Teoli D, Sanvictores T, An J. SWOT Analysis. In: StatPearls [Internet]. Treasure Island (FL): StatPearls Publishing; 2021.

2. Comitetul Economic și Social European. Impactul revoluției digitale în domeniul asistentei medicale asupra asigurărilor de sănătate. Jurnalul Oficial al Uniunii Europene, 2017; Available at: https://op. europa.eu/es/publication-detail/-/publication/82f4bd3a-e12b-11e79749-01aa75ed71a1/language-ro.

3. Kelly J, Campbell K, Gong E, Scuffham P. The Internet of Things: Impact and Implications for Health Care Delivery. J Med Internet Res. 2020;22(11):e20135.

4. Mallappallil M, Sabu J, Gruessner A, Salifu M. A review of big data and medical research. SAGE Open Med. 2020; 25(8):2050312120934839.

5. Bragazzi NL, Dai H, Damiani G, Behzadifar M, Martini M, Wu J. How Big Data and Artificial Intelligence Can Help Better Manage the COVID-19 Pandemic. Int J Environ Res Public Health. 2020; 17(9):3176.

6. Wang S, Pershing S, Lee A. Big data requirements for artificial intelligence. Curr Opin Ophthalmol. 2020;31(5):318-323.

7. Pépin JL, Bailly S, Tamisier R. Big Data in sleep apnoea: Opportunities and challenges. Respirology. 2020;25(5):486-494.

8. Okada M. Big data and real-world data-based medicine in the management of hypertension. Hypertens Res. 2021;44(2):147-153.

9. Grant-Kels JM, Sloan B, Kantor J, Elston DM. Big data and cutaneous manifestations of COVID-19. J Am Acad Dermatol. 2020;83(2):365-366.

10. Lanzer JD, Leuschner F, Kramann R, Levinson RT, Saez-Rodriguez J. Big Data Approaches in Heart Failure Research. Curr Heart Fail Rep. 2020;17(5):213-224.

11. Babić J, Babić M, Rastović P, Ćurlin M, Šimić J, Mandić K, Pavlović K. Resilience in Health and IIIness. Psychiatr Danub. 2020;32(Suppl 2):226-232

12. Moisil I, Jitaru E. E-health progresses in Romania. Int J Med Inform. 2006;75(3-4):315-21.

13. Tîrziu E, Gheorghe-Moisii M. Caracteristici de calitate ale aplicatiilor e-health. Revista Română de Informatică şi Automatică 2018; 28(2):29-40.

14. Knaup P, Benning NH, Seitz MW, Eisenmann U. eHealth and Clinical Documentation Systems. Stud Health Tehnol Inform. 2020; 274:174-188.

15. Madanian S, Norris T, Parry D. Disaster eHealth: Scoping Review. J Med Internet Res. 2020;22(10):e18310.

16. Ledwoch J, Duncker D. eHealth-smart devices revolutionizing cardiology. Herzschrittmacherther Elektrophysiol. 2020; 31(4):368-374.

17. Aardoom JJ, Loheide-Niesmann L, Ossebaard H, Riper H. Effectiveness of eHealth Interventions in Improving Treatment Adherence for Adults With Obstructive Sleep Apnea: Meta-Analytic Review. J Med Internet Res. 2020;22(2):e16972.

18. Moro Visconti R, Morea D. Healthcare Digitalization and Pay-ForPerformance Incentives in Smart Hospital Project Financing. Int J Environ Res Public Health. 2020 Mar 30;17(7):2318. 